\title{
Adaptive constraints for feature tracking
}

Article

Published Version

Hodges, K. I. (1999) Adaptive constraints for feature tracking. Monthly Weather Review, 127 (6). pp. 1362-1373. ISSN 15200493 doi: https://doi.org/10.1175/15200493(1999)127<1362:ACFFT>2.0.CO;2 Available at https://centaur.reading.ac.uk/163/

It is advisable to refer to the publisher's version if you intend to cite from the work. See Guidance on citing.

To link to this article DOI: http://dx.doi.org/10.1175/1520-

0493(1999)127<1362:ACFFT>2.0.CO;2

Publisher: American Meteorological Society

All outputs in CentAUR are protected by Intellectual Property Rights law, including copyright law. Copyright and IPR is retained by the creators or other copyright holders. Terms and conditions for use of this material are defined in the End User Agreement.

\section{www.reading.ac.uk/centaur}

\section{CentAUR}

Central Archive at the University of Reading

Reading's research outputs online 


\title{
Adaptive Constraints for Feature Tracking
}

\author{
K. I. HODGES \\ Environmental Systems Science Centre, University of Reading, Reading, United Kingdom
}

(Manuscript received 19 November 1997, in final form 23 June 1998)

\begin{abstract}
In this paper extensions to an existing tracking algorithm are described. These extensions implement adaptive tracking constraints in the form of regional upper-bound displacements and an adaptive track smoothness constraint. Together, these constraints make the tracking algorithm more flexible than the original algorithm (which used fixed tracking parameters) and provide greater confidence in the tracking results. The result of applying the new algorithm to high-resolution ECMWF reanalysis data is shown as an example of its effectiveness.
\end{abstract}

\section{Introduction}

The objective identification and tracking of atmospheric features, together with the statistical analysis of the track ensembles, provide a useful method for determining the characteristics of these features, developing climatologies for them, and studying their seasonal and interannual variability. Features in this context can be anything from regions surrounding highs, lows, or anomalies (where seasonal or longer term means are removed) in model fields, or cloud systems identified in satellite imagery.

Several automated tracking systems have been developed in the past tailored to specific applications. For example, most model-based applications have been of the symbolic token matching type, where a small number of representative points are determined, usually the maxima or minima of the prognostic field. These points are linked together based on some criteria and/or constraints suitable for the type of motion expected (Murray and Simmonds 1991; König et al. 1993; Hodges 1995). Either a simple nearest neighbor approach is used, or more sophisticated techniques based on the optimization of some cost function for the motion of all systems are used. In general all techniques have some drawbacks. The nearest neighbor approach is best suited to data that admit a small number of systems per frame; more detailed scenes can lead to problems of association, that is, the nearest neighbor might not be correct. Nearest neighbor techniques can also be dependent on the data ordering at each time step. An example of this is shown

Corresponding author address: Dr. K. I. Hodges, ESSC (Environmental Systems Science Centre), The University of Reading, Harry Pitt Building, Whiteknights, P.O. Box 238, Reading RG6 6AL, U.K. E-mail: kih@mail.nerc-essc.ac.uk in Fig. 1, where Fig. 1a is obtained using a nearest neighbor search for a certain indexing of the points in each frame, while for Fig. 1b the indexing in frame 4 has been swapped, and for Fig. 1c the indexing in frame 3 has been swapped as well. Finally, Fig. 1d is the result of applying a cost function optimization algorithm using either Fig. 1b or Fig. 1c as initialization.

Optimization-based techniques are generally better for more complicated scenes with many systems and are less dependent on the data ordering. They are also independent of the initial correspondence, that is, when systems first appear, as long as the optimization proceeds forward and backward in time so that exchanges of points between tracks can take place at the start of the tracks, subject to the token matching criteria used. However, this type of algorithm can still make mistakes, particularly in very complicated situations or when the temporal sampling is insufficient.

For cloud motion applications, the approach has predominately been to use a region matching technique such as cross correlation (Leese and Novak 1971; Schmetz et al. 1993) or to search for overlap in consecutive frames (Williams and Houze 1987; Arnaud et al. 1992). However, there is no reason why the symbolic token matching approach cannot be used for the application to cloud system tracking provided a suitable means of determining the feature points is available. For example, Endlich et al. (1971) have used the window brightness temperature minima to track cloud systems to determine cloud motion winds, while Hodges has explored two approaches to track larger-scale systems such as mesoscale cloud systems (MCS). The first approach of Hodges has used minima in the smoothed window brightness temperature field of Meteosat (Hodges and Thorncroft 1997), while the second approach has used the smoothed shape of the system to determine suitable feature points (Hodges 1998). 


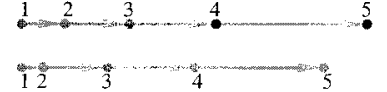

(a)

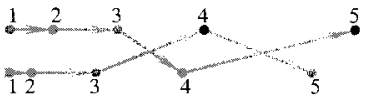

(c)

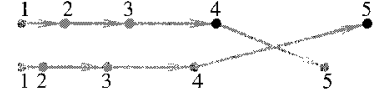

(b)

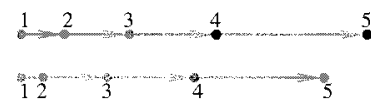

(d)
FIG. 1. Importance of feature point ordering on nearest neighbor searches: (a) result of nearest neighbor search for a particular featurepoint indexing, (b) same as (a) except indexing at frame 4 swapped, (c) same as (b) except indexing swapped at frame 3, (d) result of cost function optimization using either (b) or (c) as initialization.

The system developed for tracking and statistical analysis as described by Hodges $(1995,1996)$ is of general applicability to problems in spherical geometry (including subregions). The tracking part of this system is based on the original algorithm of Salari and Sethi (1990) adapted to work on a spherical domain (Hodges 1995). This paper reports on further enhancements to the algorithm, which make it more versatile and applicable to a wider range of data.

\section{Constraints and tracking algorithms}

All tracking algorithms impose constraints in some form both to exclude unlikely matches and to reduce the combinatorial search for matches. Fixed constraints are generally acceptable for regional studies or for studies using low-resolution data with few features. However, for global studies using high-resolution data, which may have clutter and many features, a more flexible application of constraints is useful. This will both reduce the amount of checking of likely matches and further exclude unlikely matches, which become more prevalent in higher-resolution data from both models and satellites. For example, synoptic-scale features in the Tropics will generally move more slowly and undergo possibly large changes in velocity (speed and/or direction) in a time step, due to their actual motion, measurement inaccuracy, for example, in the geo-referencing of satellite data, or due to error in data assimilation if observational data have been incorporated into a model. Systems in the midlatitude storm tracks will generally move much more quickly. For these fast-moving systems a large change in velocity (in a particular direction) is unlikely, although slow-moving systems in the storm tracks may have similar motion characteristics to a slowmoving tropical system, that is, they may vacillate in position. Here, discussion of large changes in velocity is contingent on the temporal sampling interval. For fast-moving systems sampled infrequently, large chang- es in velocity as determined by a tracking algorithm are unlikely to carry much confidence. Indeed if the temporal sampling of a field is very poor and the types of system that are to be tracked have a large velocity then the confidence in the determined tracks is likely to be low for all determined tracks, more so in a high-resolution field with many systems. There is also the liklihood of track aliasing if the time step is too large, where a coherent track is produced from essentially short-lived systems.

The simplest constraint is an upper-bound displacement, which all tracking algorithms impose in some form. In this paper the implementation of regionally varying upper-bound displacements is described for the algorithm described by Hodges (1995) for tracking on the sphere. Other types of constraints are often imposed on the tracking in the form of velocity constraints or track smoothness constraints. The former can be imposed in several ways, for example, as a compatability constraint (Wu 1995) where neighboring features have similar velocities. This has been applied in a cloud motion wind scheme where there may be several features within a small region to compare. Alternatively, mean (climatological) wind speeds may be used, if these are available, to impose some directional constraint as well as displacement. The track smoothness constraint differs from these velocity constraints in that it deals with changes in velocity rather than absolute velocities. As track smoothness is related to changes in velocity it must be measured over several frames or time steps. A minimum of three frames is required to estimate changes in velocity, although more can be used especially if changes in acceleration are used as well. The algorithm described by Hodges (1995) uses this type of constraint. This paper will describe how the track smoothness constraint can be applied adaptively along with the regionally varying upper-bound displacement constraint already mentioned. Essentially, the track smoothness constraint is allowed to vary with the local mean separation distance on a track, so that points that are close together on a track lead to a less restrictive constraint on the track smoothness while points that are far apart lead to a stricter constraint.

\section{Modified algorithm}

The original tracking algorithm, of Saleri and Sethi (1990) has been modified by Hodges (1995) to work on a spherical domain for application to geo-referenced model data. The new modifications described in this paper concern the implementation of adaptive tracking constraints in response to applications that are closer to "reality." Examples are the identification and tracking of midlatitude storms, tropical easterly waves, monsoon depressions and tropical cyclones in European Centre for Medium-Range Weather Forecasting (ECMWF) Reanalysis (ERA) data, and the identification and tracking of tropical MCSs using satellite imagery. Both of these 
sets of data will present a challenge to most tracking algorithms due to the amount of detail, some of which can be filtered or smoothed to simplify, but which will still retain a large number of possible features per frame in the form of genuine features and clutter.

The basis of the algorithm is the optimization of a cost function constructed from local track smoothness measures, with the smoothness being measured in terms of changes in direction and speed. The smoothness measure thus requires a minimum of three consecutive frames. A set of tracks is initialized, subject to the constraints, from the identified feature points for the required time sequence. Incomplete tracks are padded out with "phantom" feature points so that all tracks have the same number of points and span the length of the time series. The optimization swaps points on the tracks to give the greatest gain in smoothness and proceeds both forward and backward in time to ensure that there is no feature point ordering dependence in the final result.

To implement the adaptive constraints, several aspects of the original algorithm need to be changed and the constraints specified. The cost function to be minimized (to produce the optimal smoothness) is given by

$$
\Xi=\sum_{i=1}^{m} \sum_{k=2}^{n-1} \mathcal{D}\left(\mathbf{P}_{i}^{k-1}, \mathbf{P}_{i}^{k}, \mathbf{P}_{i}^{k+1}\right),
$$

where $\mathcal{D}\left(\mathbf{P}_{i}^{k-1}, \mathbf{P}_{i}^{k}, \mathbf{P}_{i}^{k+1}\right)$ is called the local deviation, at time step $k$, with $m$ the total number of tracks and $n$ the total number of frames or time steps. Also, $\mathbf{P}_{i}^{k}$ is the position vector in Cartesian space (a point on the sphere is represented as a unit vector in Cartesian space) for a feature point on track $i$ at time step $k$. The local deviation in the new algorithm is now defined as
$\mathcal{D}\left(\mathbf{P}_{i}^{k-1}, \mathbf{P}_{i}^{k}, \mathbf{P}_{i}^{k+1}\right)=\left\{\begin{array}{l}0 \\ \psi\left(\mathbf{P}_{i}^{k-1}, \mathbf{P}_{i}^{k}, \mathbf{P}_{i}^{k+1}\right) \\ \Psi\end{array}\right.$

if $\mathbf{P}_{i}^{k-1}$ is a phantom feature point, and $\mathbf{P}_{i}^{k}$ and $\mathbf{P}_{i}^{k+1}$ are real or phantom; if $\mathbf{P}_{i}^{k-1}, \mathbf{P}_{i}^{k}$ and $\mathbf{P}_{i}^{k+1}$ are real feature points;

otherwise, where $\psi\left(\mathbf{P}_{i}^{k-1}, \mathbf{P}_{i}^{k}, \mathbf{P}_{i}^{k+1}\right)$ is a measure of the change of speed and direction over three time steps (see below) and $\Psi$ is now a global upper bound on $\psi$ that satisfies

$$
\Psi \geq \sup _{d_{i}^{(k-1, k)}, d_{i}^{(k, k+1)} \in[0, D]} \psi_{\max }\left(d_{i}^{(k-1, k)}, d_{i}^{(k, k+1)}\right),
$$

where $\psi_{\max }\left(d_{i}^{(k-1, k)}, d_{i}^{(k, k+1)}\right)$ is the upper-bound track smoothness constraint as a function of the mean displacement over three frames, and where $d_{i}^{(k-1, k)}=$ $\left\|\mathbf{P}_{i}^{k-1} \mathbf{P}_{i}^{k}\right\|$ is the displacement distance between the point $\mathbf{P}_{i}^{k-1}$ and $\mathbf{P}_{i}^{k}$ and now is defined as

$$
\left\|\mathbf{P}_{i}^{k} \mathbf{P}_{i}^{k+1}\right\|= \begin{cases}\left\|\mathbf{P}_{i}^{k} \mathbf{P}_{i}^{k+1}\right\|_{p} & \text { if } \mathbf{P}_{i}^{k} \text { and } \mathbf{P}_{i}^{k+1} \text { are both true feature points } \\ D & \text { otherwise. }\end{cases}
$$

This is dependent on a suitable norm $\left\|\mathbf{P}_{i}^{k} \mathbf{P}_{i}^{k+1}\right\|_{p}$ (this will be the geodesic norm for a spherical domain $p=$ $g$, and the Euclidean norm $p=e$ for a Cartesian domain with no surface constraints). The parameter $D$ is a global upper bound on displacement, that is,

$$
D=\max _{j=1, N}\left(d_{\max }^{(j)}\right) ; \quad N=\text { number of regions, }
$$

where $d_{\max }^{(j)}$ is the upper-bound displacement for region $j$.

The function $\psi\left(\mathbf{P}_{i}^{k-1}, \mathbf{P}_{i}^{k}, \mathbf{P}_{i}^{k+1}\right)$ is now specified as:

$$
\begin{aligned}
\psi\left(\mathbf{P}_{i}^{k-1}, \mathbf{P}_{i}^{k}, \mathbf{P}_{i}^{k+1}\right)= & 0.5 w_{1}\left(1-\hat{\mathbf{T}}_{i}^{(k-1, k)} \cdot \hat{\mathbf{T}}_{i}^{(k, k+1)}\right) \\
& +w_{2}\left(1-\frac{2\left[\left\|\mathbf{P}_{i}^{k-1} \mathbf{P}_{i}^{k}\right\|\left\|\mathbf{P}_{i}^{k} \mathbf{P}_{i}^{k+1}\right\|\right]^{1 / 2}}{\left[\left\|\mathbf{P}_{i}^{k-1} \mathbf{P}_{i}^{k}\right\|+\left\|\mathbf{P}_{i}^{k} \mathbf{P}_{i}^{k+1}\right\|\right]}\right),
\end{aligned}
$$

where the first term measures directional similarity and the second term measures speed similarity. The unit vector $\hat{\mathbf{T}}_{i}^{(k-1, k)}$ represents the direction from point $\mathbf{P}_{i}^{k-1}$ to point $\mathbf{P}_{i}^{k}$ with $\left\|\mathbf{P}_{i}^{k-1} \mathbf{P}_{i}^{k}\right\|$ as defined above. Both $\hat{\mathbf{T}}_{i}^{(k-1, k)}$ and $\left\|\mathbf{P}_{i}^{k-1} \mathbf{P}_{i}^{k}\right\|$ will depend on the domain of the application, here taken as a spherical domain (see Hodges 1995 for further details). Note the addition of the factor 0.5 in the first term compared with the definition in previous descriptions (Salari and Sethi 1990; Hodges 1995). This ensures that provided $w_{1}+w_{2}=1$, then $\psi\left(\mathbf{P}_{i}^{k-1}, \mathbf{P}_{i}^{k}, \mathbf{P}_{i}^{k+1}\right) \in[0,1]$ for all changes in direction $\left(\left|\hat{\mathbf{T}}_{i}^{(k-1, k)} \cdot \hat{\mathbf{T}}_{i}^{(k, k+1)}\right| \leq 1 \Rightarrow 0 \leq 1-\hat{\mathbf{T}}_{i}^{(k-1, k)} \cdot \hat{\mathbf{T}}_{i}^{(k, k+1)} \leq 2\right)$ and speed.

It only remains to specify the form the adaptive constraints take and to outline the modified algorithm that implements them. The upper-bound displacements are 
specified by choosing the extent of the regions. On a sphere this will typically be in terms of lat-long boxes or as zones. For example, for a global study of synopticscale systems using relative vorticity, the regions might be specified as zones, one for the Tropics and one each for the Northern and Southern Hemisphere extratropics (more could be specified if required). An upper-bound displacement for each region is then assigned. In the example just mentioned, this would result in a lower value for the Tropics than for the extratropics. The variable upper bound displacement function is defined as

$$
d_{\max }\left(\mathbf{P}_{i}^{k-1}, \mathbf{P}_{i}^{k}\right)= \begin{cases}D & \text { if either } \mathbf{P}_{i}^{k-1}, \mathbf{P}_{i}^{k} \text { are phantom points. } \\ d_{\max }^{(j)} & \text { if both } \mathbf{P}_{i}^{k-1}, \mathbf{P}_{i}^{k} \in \text { region } j . \\ 0.5\left(d_{\max }^{j}+d_{\max }^{l}\right) & \text { if } \mathbf{P}_{i}^{k-1} \in \text { region } j \text { and } \mathbf{P}_{i}^{k} \in \text { region } l \text { or vice versa. }\end{cases}
$$

More complex functions might be designed, for example, a continuous function that increases linearly from the equator to the poles might be used, but the function described above is probably the simplest and easiest to apply given general regions with boundaries in longitude as well as latitude.

The specification of the adaptive track smoothness constraint is less straightforward as it will depend to some extent on the perception of the type of motion present in the data. In practice, in the absence of clutter and ambiguity over matching in consecutive frames, that is, when there is only one possible match, then the best match will be the nearest neighbor in the next frame irrespective of track smoothness. However, this does not necessarily mean it is a correct match if the change in velocity (speed and direction) is unrealistic for the type of systems being tracked. It is more likely to be correct, irrespective of change in velocity, if the points are close together either because the system is slow moving or

$$
\mathcal{D}_{\max }\left(\mathbf{P}_{i}^{k-1}, \mathbf{P}_{i}^{k}, \mathbf{P}_{i}^{k+1}\right)=\left\{\begin{array}{l}
\psi_{\max }\left(d^{(k-1, k)}, d^{(k, k+1)}\right) \\
\Psi
\end{array}\right.
$$

As with the variable upper-bound displacement, any suitable function can be used but here the piecewise linear function shown in Fig. 2 which is relatively simple and cheap to compute is used. This form for the adaptive smoothness has a large value for small average displacement distances over three time steps and decreases piecewise linearly to a smaller value for large average displacement distances over three time steps in accordance with the discussion above.

The cost function is minimized locally in time subject to the constraints using an adapted form of the modified Greedy exchange algorithm (MGEA) proposed by Sethi and Jain (1987) and adapted for incomplete tracks by Saleri and Sethi (1990). This is described in the next section. because the temporal sampling rate is high. On the other hand, for widely separated points, either because the system is fast moving or because the sampling rate is low (but within the upper-bound displacement), we will have a low confidence in large changes in velocity, and more particularly, in direction. For example, we would not expect a midlatitude storm moving within the storm track at a speed representative of such systems to double back on itself over the period of three time steps. On the other hand, we might expect a system that is vacillating to "wobble" about in position and maybe to have a large relative change in speed. So a less restrictive track smoothness constraint is required if the system is slow moving and a more restrictive constraint is required if it is fast moving. The form of the adaptive constraint chosen is shown in Fig. 2.

The function actually used for the upper-bound track smoothness is defined as

if $\left(\mathbf{P}_{i}^{k-1}, \mathbf{P}_{i}^{k}, \mathbf{P}_{i}^{k+1}\right)$ are all real feature points. otherwise.

\section{Operation of the MGEA with adaptive constraints}

The MGEA is initialized by first constructing a set of tracks from the available feature points using a nearest neighbor search and subject to the regional upperbound displacements. Incomplete tracks are padded out with phantom feature points. In the original algorithm a set of tracks was required to be initialized with only phantom points to facilitate the exchange of features for tracks exceeding the upper-bound track smoothness, so that all final tracks after the optimization of the cost function satisfy the smoothness constraint. The new algorithm requires substantially fewer of these since a new step is introduced that processes the tracks before each forward or backward iteration to ensure that none of 


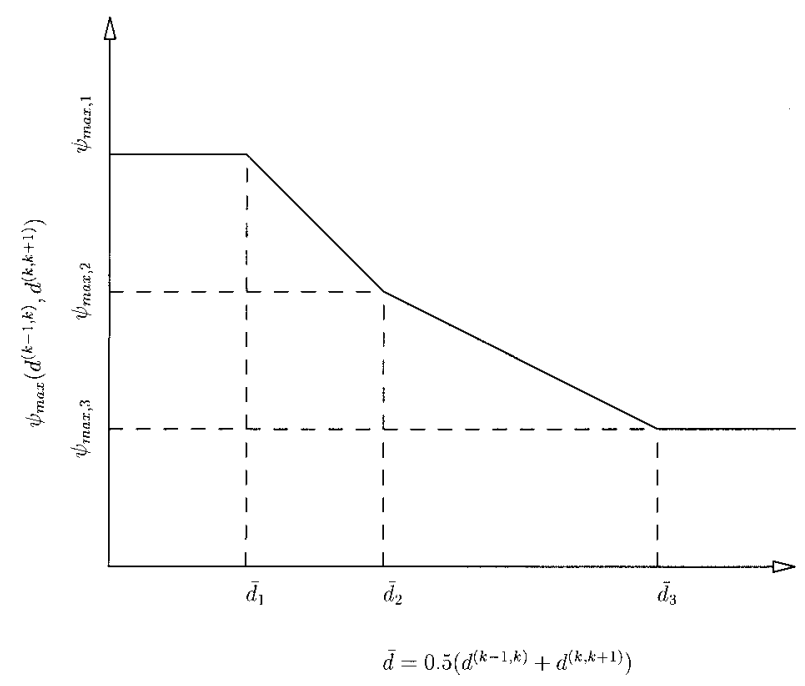

FIG. 2. Schematic of a piecewise linear adaptive track smoothness constraint as a function of local mean displacement distance $\left[\left(\mathbf{P}_{i}^{k-1}\right.\right.$, $\left.\mathbf{P}_{i}^{k}, \mathbf{P}_{i}^{k+1}\right)$ all real feature points].

them exceed the local track smoothness constraint, either as a fixed constraint applied globally or as an adaptive constraint. Tracks are broken up if the constraints are violated anywhere. This step is essential for applying the adaptive track smoothness constraint, but also has the added benefit in that the optimization process is generally faster for fixed constraints, although for the adaptive constraints the extra function evaluations can slow down the optimization. The optimization is iterative, proceeding both forward and backward in time and swapping those pairs of points on tracks that provide the greatest gain in track smoothness subject to the constraints.

An outline of the MGEA with adaptive constraints is described in appendix $\mathrm{A}$, in a similar form to the original Sethi and Jain (1987) algorithm so that it is apparent how the new aspects of the algorithm fit into the original algorithm.

In practice the algorithm can get stuck when in fact it should have terminated. This can occur when there is a conflict between a forward and backward iteration on a small number of tracks. This will depend on the values set for the weights $w_{1}$ and $w_{2}$ in the local smoothness function. An example of this is shown in Fig. 3. To ensure a termination occurs, a maximum number of forward and backward iterations is specified, if the algorithm exceeds this limit it terminates with one final forward iteration. In practice most of the work is done in the first two forward and backward iterations, so the limit is usually set at 3 .

As mentioned in the introduction this algorithm is not perfect and will make mistakes; in fact it can construct tracks that satisfy the constraints and that represent the minimum of the cost function but that are incorrect. The flexibility of adaptive constraints can help to reduce the mistakes provided the variation in
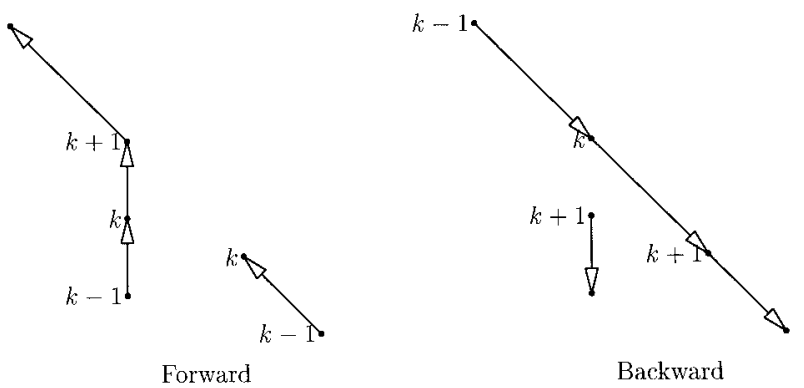

FIG. 3. Example of tracks with ambiguous track smoothness.

the constraints is chosen wisely. This highlights the point that some form of sensitivity study should be performed on the constraints before the final processing is performed. If observational data exist for the features of interest, then these can be used as a basis for choosing the constraints.

There is one last point concerning long time series. Because the optimization process can be time consuming if there are a large number of features per time step, the implementation of the algorithm is such that it can be applied to overlapping sections of the time series. Tracks from the sections are then spliced together to form the final track ensemble for the whole time series. In previous use, the overlap was set at two time steps (two time steps in common between two sections), but it has since been noticed that this can lead to incorrect matches when the tracks are spliced together. The overlap should be at least three time steps to avoid this problem.

\section{Application to the ECMWF reanalysis}

To provide an example of the algorithm in action, it has been applied to data from the ERA project (Gibson et al. 1997). The ERA data are based on the integration of the integrated forecast system with observational data assimilated using optimal interpolation. The field chosen for this example is the relative vorticity at $850 \mathrm{hPa}$. The data is provided at a spectral resolution of T106 and time step of $6 \mathrm{~h}$, and has been interpolated to pressure levels. The vorticity field at this resolution has a large amount of detail representing a large range of spatial and temporal scales, relating to both synoptic systems as well as higher-frequency transients and smaller spatial scales. It is this amount of detail that makes the field one of the most challenging to study with tracking systems as there is much scope for making mistakes at least at the temporal sampling rate of $6 \mathrm{~h}$ (there are likely to be several possible matches within the chosen search radius). Filtering both in space and time can remove some of this ambiguity before the tracking system is applied, but for this example no filtering has been performed to see how the new system performs for such complicated situations. The vorticity is also the best field for look- 
TABLE 1. Adaptive constraints used for the example. The last row indicates the allowed maximum speed in each zone.

\begin{tabular}{|c|c|c|c|c|c|c|}
\hline \multicolumn{7}{|c|}{ Zonal upper-bound displacements } \\
\hline Zones & & 1 & 2 & 3 & 4 & 5 \\
\hline Lower $(\mathrm{d}$ & & -90.0 & -30.0 & -20.0 & 20.0 & 30.0 \\
\hline Upper (de & & -30.0 & -20.0 & 20.0 & 30.0 & 90.0 \\
\hline$d_{\max }^{\mathrm{j}}(\mathrm{deg})$ & & 5.0 & 4.0 & 3.5 & 4.0 & 5.0 \\
\hline Max. spe & $\left.\mathrm{m} \mathrm{s}^{-1}\right)$ & 25 & 20 & 18 & 20 & 25 \\
\hline \multicolumn{7}{|c|}{ Adaptive track smoothness } \\
\hline $\bar{d}(\mathrm{deg})$ & 0.0 & 1.0 & 2.0 & 5.0 & \multicolumn{2}{|c|}{$>5.0$} \\
\hline$\Psi_{\max }(\bar{d})$ & 1.0 & 1.0 & 0.2 & 0.08 & \multicolumn{2}{|c|}{0.08} \\
\hline
\end{tabular}

ing at tropical activity like easterly waves (Reed et al. 1988) and for tropical activity like monsoon depressions and tropical cyclones. The example presented below is for the period of May-September from 1989 and for the region of the Northern Hemisphere Indian Ocean and western Pacific. The aim is to identify and track monsoon depressions over India and the important systems in the western Pacific, that is, tropical storms. The identification starts by applying a threshold of $3.0 \times 10^{-5} \mathrm{~s}^{-1}$ so that all data above this threshold are retained and searched for maxima (cyclonic systems). This is a somewhat higher value than is usually used at lower resolutions, but at this higher resolution the systems of interest will appear more intense than, say, at T42 resolution. However, a substantial amount of clutter is still retained, particularly around the orography so the choice of the tracking parameters must be chosen carefully. This threshold is suitable for the monsoon depressions and tropical storms that we are interested in; however, this is likely to need reducing for easterly waves that are much weaker systems, with the consequence that a lot more clutter (short-lived systems) will be present. Easterly waves present problems in another way in data of this type in that they have the propensity to develop multiple centers that make it much more difficult to obtain coherent tracks. This happens much less often in more intense systems, making them easier to track. The application to easterly waves is still under consideration, but it has been found that reducing the resolution by smoothing onto a lower-resolution grid makes the tracking of the synoptic-scale waves easier.

The tracking parameters that have been used are $w_{1}$ $=0.2, W_{2}=0.8$ for the local smoothness measure weights. The adaptive constraints have been chosen following a limited sensitivity study and are specified in Table 1. The guide to choosing these parameters is that they should be as restrictive as is compatible with the type of system to be tracked, taking account of measurement error in the data. The choice of the parameters in Table 1 satisfies this criteria for the tropical systems that are of interest here and for the time sampling of the data $(6 \mathrm{~h})$.

The values for the upper-bound displacements can be interpreted as a maximum speed. The values for the maximum allowed speed in each zone are given in Table 1. The values of the track smoothness constraint can be interpreted in terms of the maximum allowed change in direction (for fixed speed) or change in speed (for fixed direction). Of course, for changes in both direction and speed neither maximum is attained. For example, for an average displacement over three frames of less than or equal to $1.0^{\circ}$ (geodesic) the maximum allowed track smoothness is unity, which means all changes in speed and direction are allowed subject to this average displacement. On the other hand, for average displacements greater than $5^{\circ}$ (geodesic) the maximum allowed track smoothness is 0.08 . If there is no change in speed this is equivalent to a maximum allowed change in direction of $80^{\circ}$, if there is no change in direction this is equivalent to a change in speed of $60 \%$. The form of the constraint on track smoothness decreases from the former to the latter as average displacement distance increases. These values were acceptable for the application here. Ideally the constraint values can be chosen from observations if available, although care must be taken to ensure that the observations are numerous enough to provide statistically reliable values. In practice, although we find observed system velocities are a good guide for setting the constraints, there are always those systems that will not satisfy the constraints resulting in a tracking error. This is particularly the case with ERA at high resolution using the vorticity field where limitations in the observed data that are assimilated into the model integration may cause very sudden changes in motion that may not be typical of the actual systems of interest; an insufficient sampling rate can make the problem worse. This sudden change in motion may manifest itself as both a large change in displacement distance and/or a large change in direction, so to allow for this in the tracking constraints by relaxing them sufficiently may result in errors occuring elsewhere. Inevitably there is a trade-off in choosing the constraints such that the majority of systems are tracked correctly at the expense that some systems undergoing large changes in velocity are incorrectly tracked. For a less cluttered field or for a larger data sampling rate some relaxation of the track smoothness constraints is possible so that the systems that do exhibit these large changes in velocity are tracked correctly. This leads back to the idea of applying some filtering to remove the clutter; certainly reducing the resolution, for example, from T106 to T42 (synoptic-scale systems are still resolved), allows the constraints to be relaxed and the tracking to make fewer mistakes. The constraints can also be better chosen if the focus is on particular types of systems and/or particular geographical regions. For example, tropical storms such as typhoons and hurricanes are relatively large-scale, intense, tropical phenomena predominately found in the oceanic regions. Focusing just on this type of system it is found that the constraints can be relaxed considerably. However, for the 

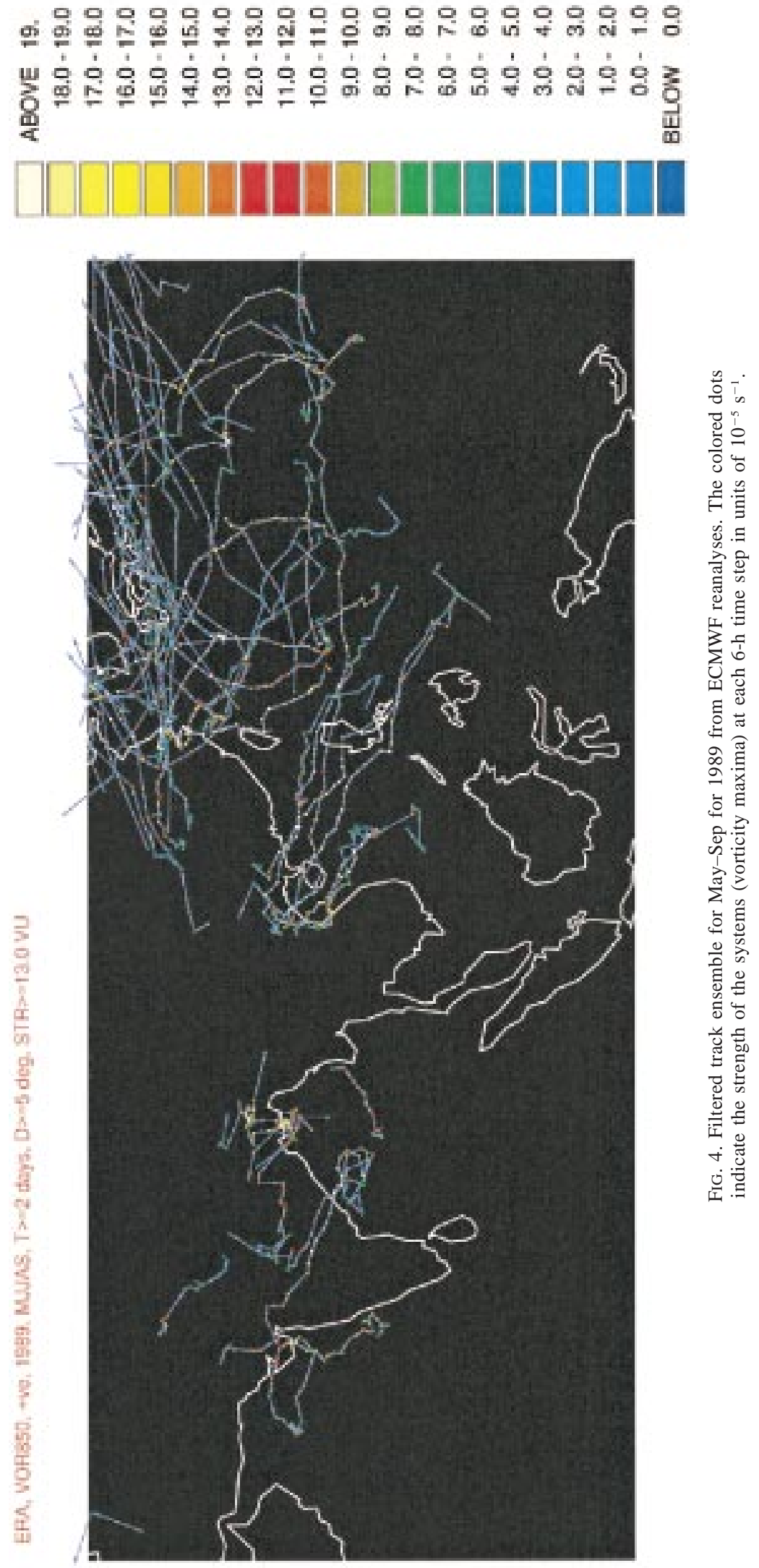


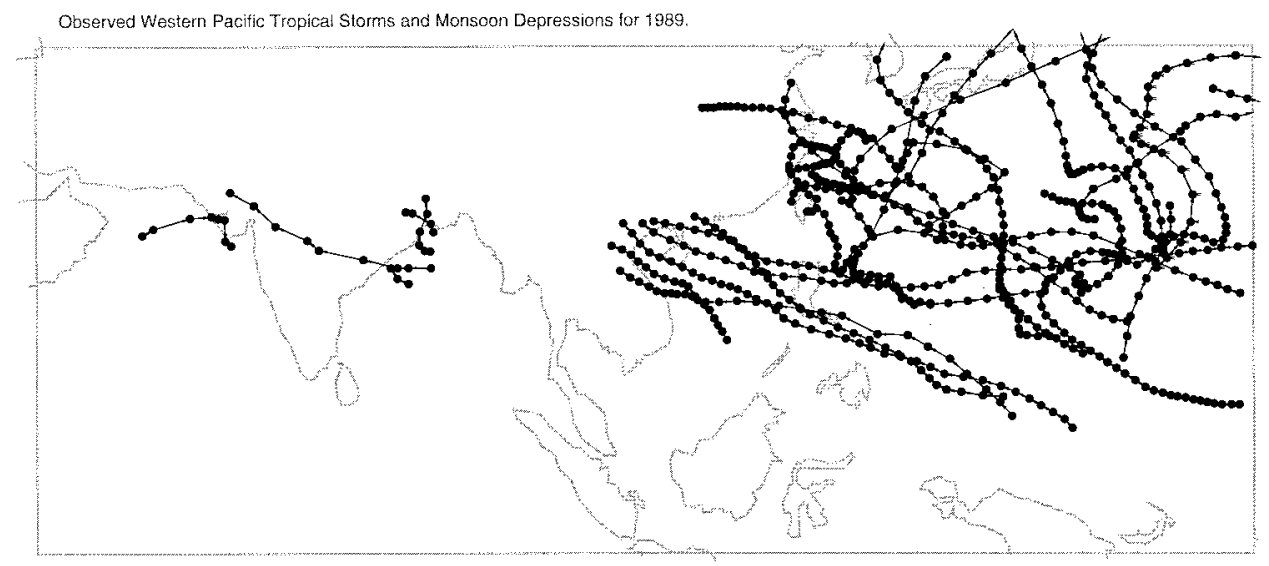

FIG. 5. The tracks of observed tropical storms and monsoon depressions for May-Sep 1989. Observations are 6 hourly for the western Pacific tropical storms and 12 hourly for the monsoon depressions. The observed monsoon data are available only for the mature phase of the system from the Mausam (Gupta et al. 1990) journal.

monsoon depressions that are generally associated with land and are weaker types of system, we find the constraints need to be chosen more carefully, particularly if there is neighboring orography that can generate spurious systems when reduction to pressure levels is performed.

Figure 4 shows the resultant track ensemble for the May-September period of 1989. The tracks have been filtered to remove tracks that last less than 2 days and travel less than $5^{\circ}$ (about $500 \mathrm{~km}$ ). Also, systems have to attain a strength of at least $13.0 \times 10^{-5} \mathrm{~s}^{-1}$ to be retained. This removes the tracks for stationary and short-lived systems leaving only the coherent longerlived system tracks, which are likely to be associated with rain bearing systems.

Figure 4 shows several aspects of interest associated with the Asian monsoon. There are several systems generated in the Bay of Bengal that move northward into Bangladesh. In this particular year there are also several systems that propagate inland from the Bay of Bengal over the Eastern Ghats. These are all systems associated with the main phase of the monsoon. However, on the western side of India systems can often be observed that develop in the southern Arabian sea and propagate northward along the coast of India. These are associated with the monsoon onset. In this particular year one of these systems generated in situ to the west of Goa can be seen meandering northward to the Gujarat region and then out into the Arabian sea.

In the western Pacific there are several very intense systems associated with tropical storms (although other fields would need to be examined to clearly identify them as typhoons). There is clearly a contrast between those systems that propagate predominantly in a zonal direction, for example, those that penetrate into Southeast Asia (particularly North Vietnam) and those that propagate predominately northward into the Pacific storm track. It has been proposed that the remnants of the tropical storms that frequent Southeast Asia may cross the landmass and act as precursors to the storms that develop in the Bay of Bengal (Saha et al. 1981). However, in Fig. 4 this is not apparent, with all the systems that propagate into Southeast Asia (Vietnam) disappearing there, although in other years (not shown) there is a hint of this behavior occurring. There may be several reasons for this lack of apparent mobility across the landmass. The systems probably become very weak and may not even be closed systems, so that the approach to identification used here will not identify them, particularly with the relatively high threshold used here. Even reducing the threshold to a lower level may not identify them very well since, as mentioned in the brief discussion above on tracking African easterly waves using the relative vorticity data, the field becomes very noisy at low intensity levels with a propensity of even coherent systems to develop multiple centers, making their tracking very difficult. However, since they are synoptic-scale systems, filtering of the data offers some hope that if this type of motion occurs and the model is resolving it sufficiently, they may still be identified and tracked. This will require further investigation.

These results can be compared with the observed storms for this period, although this type of comparison is a function of how well ERA represents the storms and how well the tracking algorithm has performed. The main storms for this period are shown in Fig. 5.

Overall, the main western Pacific storms appear to have been picked out reasonably well, although there are differences that may be due to either how well ERA represents the storms or to mistakes by the tracking algorithm. Another reason for differences is the way the storms have been identified. In Fig. 4 simple criteria 


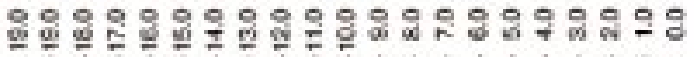

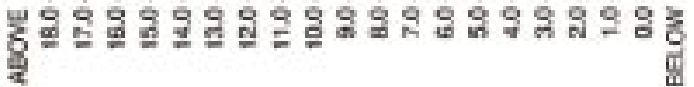
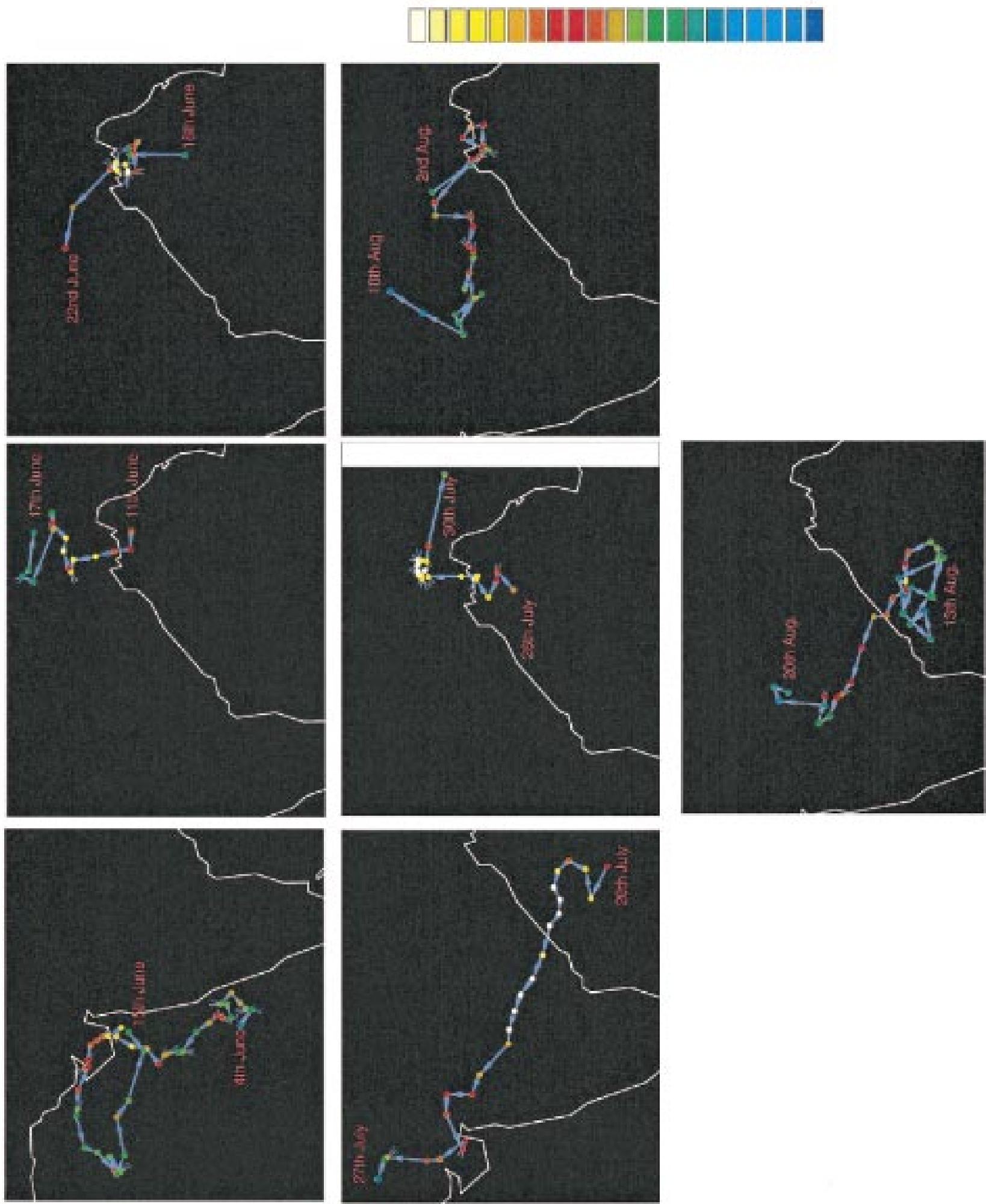


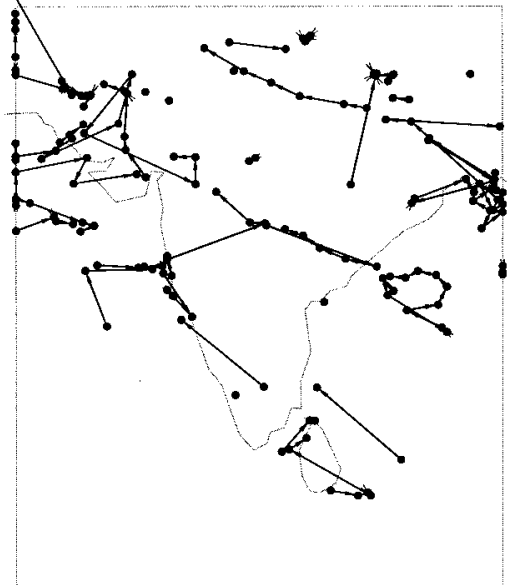

(a) Initialization for $\mathrm{d}_{\max }=6.0 \mathrm{deg}$.

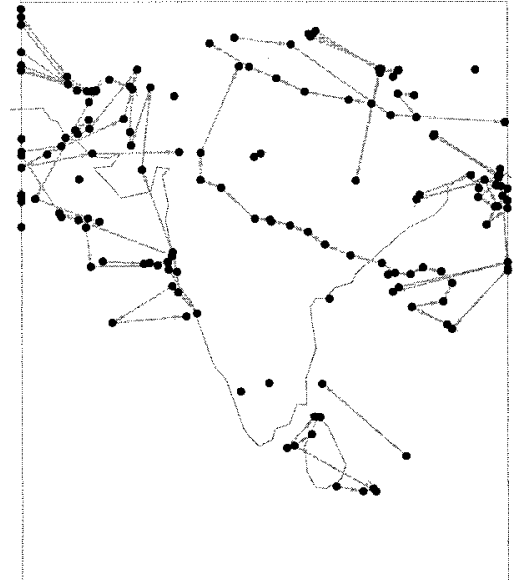

(b) After optimization for $\mathrm{d}_{\max }=6.0 \mathrm{deg}$.

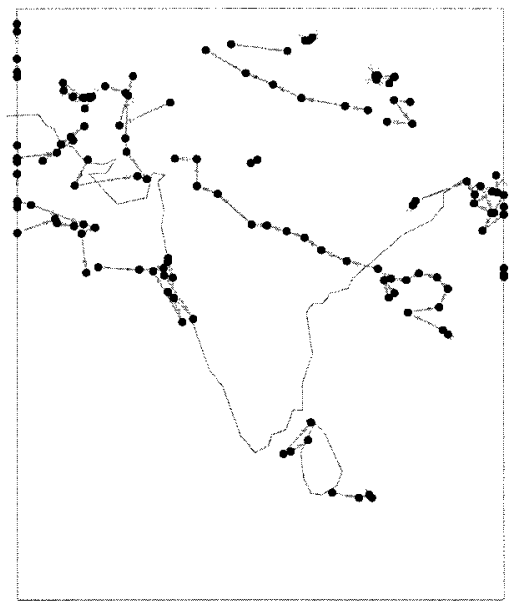

(c) Initialization for $d_{\max }=3.0 \mathrm{deg}$.

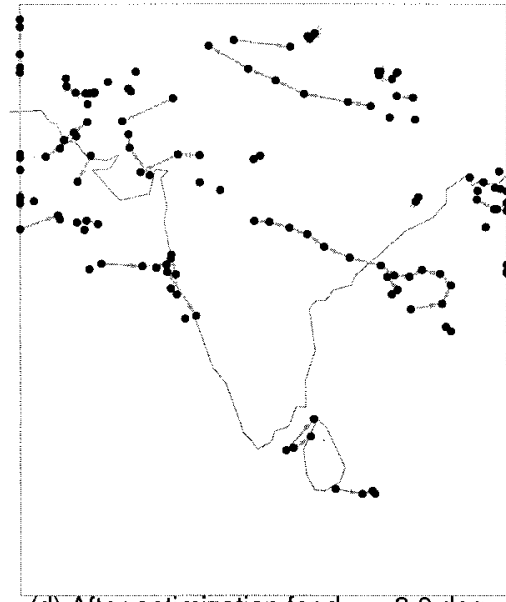

(d) After optimization for $\mathrm{d}_{\text {max }}=3.0 \mathrm{deg}$.

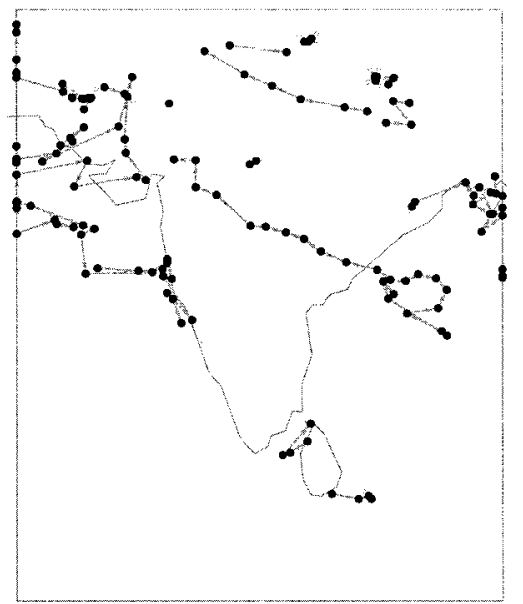

(e) Initialization for adaptive constraints.

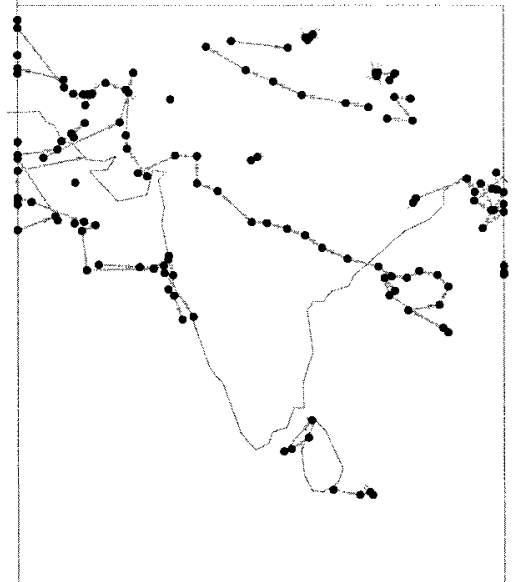

(f) After optimization with adaptive constraints.

FIG. 7. Comparison using fixed constraints with adaptive constraints. (a) Nearest neighbor initialization with $d_{\max }=6^{\circ}$; (b) after optimization with $d_{\max }=6^{\circ}, \psi_{\max }=1.0$; (c) nearest neighbor initialization with $d_{\max }=3^{\circ}$; (d) after optimization with $d_{\max }=3^{\circ}, \psi_{\max }=0.1$; (e) nearest neighbor initialization with zonal $d_{\max }$; (f) after optimization with zonal $d_{\max }$ and adaptive track smoothness constraints.

based on the attained strength, displacement distance, and lifetime are used as a filter after tracking, while the storms in Fig. 5 are generally identified using alternative objective criteria, such as wind speed, together with satellite and radar observations. A better result may be possible by using different constraints over land than over the ocean. The reason for this is that there is more noise over land associated with the orography, and reduction of the data to pressure levels, than over the oceans.

The monsoon depressions obtained from ERA and associated with the observed systems are shown in more detail in Fig. 6. These correspond reasonably well with the mature phase of the depressions when compared with the observed storms. However, for 1989 only five main depressions were observed whereas we have identified seven strong storms in the ERA data, two of which (26-30 July, 18-22 August) do not appear to have been classified as monsoon depressions.

It is clear from these results that in general the motion of tropical systems as modeled by the reanalyses (and also observed) is not very smooth, in keeping with the meandering nature of these systems, although some of the lack of smoothness may be due to the way observational data is assimilated into the model integration. However, the new algorithm has successfully tracked the important systems even though there is a significant amount of clutter, both orographically induced as well as model induced.

One final comparison is made to highlight the ef- 


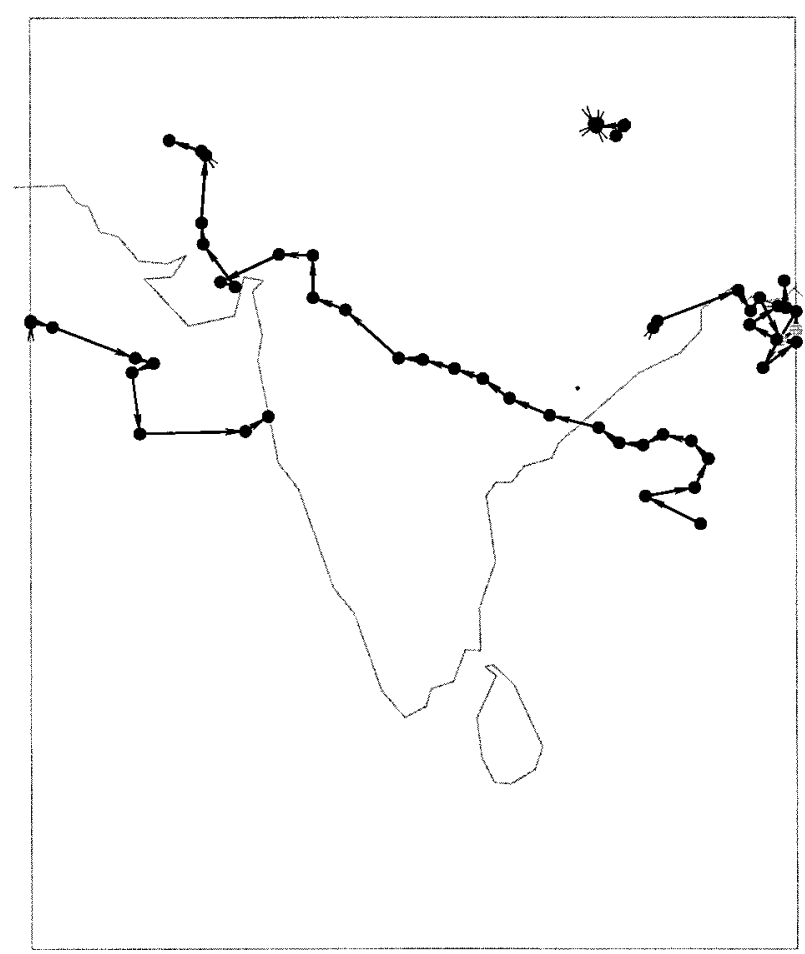

FIG. 8. As in Fig. 7f but for tracks with lifetimes less than 2 days (eight time steps) removed.

fectiveness of using the adaptive constraints. This comparison takes the form of running the tracking code for a set of fixed but lax constraints, a set of fixed but strict constraints, and for the adaptive constraints. This was done for the frame interval of 320-352 over India, that is, the period and region where the monsoon depression of 20-27 July occured. The results are shown in Fig. 7, where the dots represent feature points identified in the frame interval. This clearly shows the degree of clutter. The top row indicates the nearest neighbor initialization for (a) the fixed upper-bound displacement of $6.0^{\circ}$ (a lax constraint), (c) the fixed upperbound displacement of $3.0^{\circ}$ (a relatively strict constraint), and (e) the zonal upper-bound displacements discussed above. The lower row shows the result of applying the optimization for (b) the lax constraint using $\psi_{\max }=1.0$, that is, unconstrained, for (d) the strict constraints with $\psi_{\max }=0.1$ and (f) for the adaptive constraints. The superiority of the method with adaptive constraints is more obvious if those systems with lifetimes less than 2 days (eight time steps) are removed. Figure 8 shows this for the results using the adaptive constraints, where we see the monsoon depression previously identified.

\section{Conclusions}

A new version of an existing tracking algorithm has been described that implements regional upper-bound displacements and adaptive track smoothness constraints. This provides a more flexible algorithm that can be applied with confidence to a wide range of applications. During the application of these techniques to the ECMWF reanalyses it was found that high-frequency variation over and around the orography can lead to problems of association particularly for the monsoon systems propagating along the monsoon trough close to the Tibetan Plateau as a result of the reduction of the data on model levels to pressure levels. One possibility of reducing this problem is to apply a high-pass filter to the data; this will be considered in the future. This might also help with the tracking of those weak systems that act as precursors to the development of monsoon depressions. Alternatively, applying different constraints to oceanic and continental regions may be useful.

The algorithm is currently being used to diagnose the variability and nature of mobile systems in the ECMWF reanalysis data for the Northern and Southern Hemisphere storm tracks, tropical easterly waves, and for the monsoon depressions as illustrated in the example presented.

\section{APPENDIX}

\section{A New Modified Greedy Exchange Algorithm}

1. Initialize. Initialize tracks using nearest neighbor search for all $h_{k}$ feature points $\left(h_{k}\right.$ is the number of feature points at time step $k), k=1, \ldots, n$ subject to the regional upper-bound displacement constraint $d_{\max }\left(\mathbf{P}^{k-1}, \mathbf{P}^{k}\right)$. Pad incomplete tracks with phantom points so that all tracks span $n$ frames.

\section{Optimization.}

a. Set forward and backward iteration flags to TRUE.

$b$. Forward iteration.

1) Process all tracks forward in time once. If $\mathcal{D}\left(\mathbf{P}_{i}^{k-1}, \mathbf{P}_{i}^{k}, \mathbf{P}_{i}^{k+1}\right)>\mathcal{D}_{\max }\left(\mathbf{P}_{i}^{k-1}, \mathbf{P}_{i}^{k}, \mathbf{P}_{i}^{k+1}\right)$, then break track and create a new track at end of track database for track piece from $k+1$ to $n$. Thus all tracks and new tracks formed from broken tracks are tested.

2) If forward iteration flag is TRUE do forward exchange iteration. For each time step $k=$ $2, \ldots, n-1$ :

(i) Initialize $g_{\max }=0$.

(ii) Calculate the gain $g_{i, j}^{k}$ for all tracks $i$ ( $i$ $=1, \ldots, m-1)$ and $j(j=i+1, \ldots$, $m)$; provided $d\left(\mathbf{P}_{i}^{k, k+1}, \quad \mathbf{P}_{j}^{k, k+1}\right) \leq$ $d_{\max }\left(\mathbf{P}_{i}^{k, k+1}, \mathbf{P}_{j}^{k, k+1}\right)$ and $d\left(\mathbf{P}_{j}^{k, k+1}, \mathbf{P}_{i}^{k, k+1}\right) \leq$ $d_{\max }\left(\mathbf{P}_{j}^{k, k+1}, \mathbf{P}_{i}^{k, k+1}\right)$ as:

$$
\begin{aligned}
g_{i, j}^{k}= & \mathcal{D}\left(\mathbf{P}_{i}^{k-1}, \mathbf{P}_{i}^{k}, \mathbf{P}_{i}^{k+1}\right) \\
& +\mathcal{D}\left(\mathbf{P}_{j}^{k-1}, \mathbf{P}_{j}^{k}, \mathbf{P}_{j}^{k+1}\right) \\
& -\mathcal{D}\left(\mathbf{P}_{i}^{k-1}, \mathbf{P}_{i}^{k}, \mathbf{P}_{j}^{k+1}\right) \\
& -\mathcal{D}\left(\mathbf{P}_{j}^{k-1}, \mathbf{P}_{j}^{k}, \mathbf{P}_{i}^{k+1}\right) .
\end{aligned}
$$


Assign $g_{\max }=g_{i, j}^{k}$; if $g_{i, j}^{k}>g_{\max }$ and $\mathcal{D}\left(\mathbf{P}_{i}^{k-1}, \mathbf{P}_{i}^{k}, \mathbf{P}_{j}^{k+1}\right) \leq \mathcal{D}_{\text {max }}\left(\mathbf{P}_{i}^{k-1}, \mathbf{P}_{i}^{k}, \mathbf{P}_{j}^{k+1}\right)$ and $\mathcal{D}\left(\mathbf{P}_{j}^{k-1}, \mathbf{P}_{j}^{k}, \mathbf{P}_{i}^{k+1}\right) \leq \mathcal{D}_{\max }\left(\mathbf{P}_{j}^{k-1}, \mathbf{P}_{j}^{k}\right.$, $\left.\mathbf{P}_{i}^{k+1}\right)$. Save track indices for current $g_{i, j}^{k}$.

(iii) Swap points $\mathbf{P}_{i}^{k+1}, \mathbf{P}_{j}^{k+1}$ on tracks $i$ and $j$ corresponding to final $g_{\max }$.

3) If a swap occurred during forward iteration set backward iteration flag to TRUE and return to step 2.b.2), or else set forward iteration flag to FALSE and check for completion of algorithm.

c. Backward iteration

1) Process all tracks backward in time once. If $\mathcal{D}\left(\mathbf{P}_{i}^{k+1}, \mathbf{P}_{i}^{k}, \mathbf{P}_{i}^{k-1}\right)>\mathcal{D}_{\max }\left(\mathbf{P}_{i}^{k+1}, \mathbf{P}_{i}^{k}, \mathbf{P}_{i}^{k-1}\right)$, then break track and create a new track at end of track database for track piece from 1 to $k-1$.

2) If backward iteration flag is TRUE, do a backward iteration. For each time step $k=n-$ $1, \ldots, 2$ :

(i) As for forward iteration but now exchanging points $\mathbf{P}_{i}^{k-1}, \mathbf{P}_{j}^{k-1}$.

3) If a swap occured during backward iteration, set forward iteration flag to TRUE and return to step 2.c.2), or else set backward iteration flag to FALSE and check for completion of algorithm.

d. Termination check. If both forward and backward iteration flags are FALSE, then end, otherwise repeat iteration for the iteration flags that are TRUE, starting at step 2.b for forward, and step 2.c for backward.

3. Final check. Perform a final backward and forward check on the track smoothness.

\section{REFERENCES}

Arnaud, Y., M. Desbois, and J. Maizi, 1992: Automatic tracking and characterization of African convective systems on meteosat pictures. J. Appl. Meteor., 31, 443-453.

Endlich, R. M., D. E. Wolf, D. J. Hall, and A. E. Brain, 1971: Use of a pattern recognition technique for determining cloud motions from sequences of satellite photographs. J. Appl. Meteor., 10, $105-117$.

Gibson, J. K., P Kållberg, S. Uppala, A. Hernandez, A. Nomura, and E. Serrano, 1997: ERA description. ECMWF RE-Analysis Project Report Series, No. 1, 71 pp. [Available from ECMWF, Shinfield Park, Reading RG2 9AX, United Kingdom.]

Gupta, G. R., D. S. Desai, and N. C. Biswas, 1990: Monsoon season (June-September 1989). Mausam, 41, 505-520.

Hodges, K. I., 1995: Feature tracking on the unit sphere. Mon. Wea. Rev., 123, 3458-3465.

—, 1996: Spherical nonparametric estimators applied to the UGAMP GCM integration for AMIP. Mon. Wea. Rev., 124, 2914-2932.

_ 1998 : Feature-point detection using distance transforms: Application to tracking tropical convective complexes. Mon. Wea. Rev., 126, 785-795.

— , and C. D. Thorncroft, 1997: Distribution and statistics of African mesoscale convective weather systems based on the ISCCP METEOSAT imagery. Mon. Wea. Rev., 25, 2821-2837.

König, W., R. Sausen, and F. Sielmann, 1993: Objective identification of cyclones in GCM simulations. J. Climate, 6, 2217-2231.

Leese, J. A., and C. S. Novak, 1971: An automated technique for obtaining cloud motion from geosynchronous satellite data using cross correlation. J. Appl. Meteor., 10, 118-132.

Murray, R. J., and I. Simmonds, 1991: A numerical scheme for tracking cyclone centres from digital data. Part 1: Development and operation of the scheme. Aust. Meteor. Mag., 39, 155-166.

Reed, R. J., A. Hollingsworth, W. A. Heckley, and F. Delsol, 1988: An evaluation of the performance of the ECMWF operational system in analyzing and forecasting easterly wave disturbances over Africa and the tropical Atlantic. Mon. Wea. Rev., 116, 824865 .

Saha, K., F. Sanders, and J. Shukla, 1981: Westward propogating precussors of monsoon depressions. Mon. Wea. Rev., 109, 330343 .

Salari, V., and I. K. Sethi, 1990: Feature point correspondence in the presence of occlusion. IEEE Trans. PAMI, 12, 87-91.

Schmetz, J., K. Holmlund, J. Hoffman, B. Strauss, B. Mason, V. Gaertner, A. Koch, and L. van de Berg, 1993: Operational cloudmotion winds from meteosat infrared images. J. Appl. Meteor., 32, 1206-1225.

Sethi, I. K., and R. Jain, 1987: Finding trajectories of feature points in a monocular image sequence. IEEE Trans. PAMI, 9, 56-73.

Williams, M., and R. A. Houze Jr., 1987: Satellite observed characteristics of winter monsoon cloud clusters. Mon. Wea. Rev., 115, 505-519.

Wu, Q. X., 1995: A correlation-relaxation-labelling framework for computing optical flow-Template matching from a new perspective. IEEE Trans. PAMI, 17, 843-853. 